\title{
Regenerative Medicine and the Biliary Tree
}

\author{
Thiago M. De Assuncao, MS ${ }^{1,2, *}$ Nidhi Jalan-Sakrikar, PhD ${ }^{1,2, *}$ Robert C. Huebert, MD ${ }^{1,2,3}$
}

${ }^{1}$ Division of Gastroenterology and Hepatology, Mayo Clinic and Foundation, Rochester, Minnesota

2 Gastroenterology Research Unit, Mayo Clinic and Foundation, Rochester, Minnesota

${ }^{3}$ Center for Cell Signaling in Gastroenterology, Mayo Clinic and

Foundation, Rochester, Minnesota

Address for correspondence Robert C. Huebert, MD, Gastroenterology Research Unit, Mayo Clinic, 200 First Street SW, Rochester, MN 55905 (e-mail: huebert.robert@mayo.edu).

Semin Liver Dis 2017;37:17-27.

\begin{abstract}
Keywords

- cholangiocytes

- regenerative medicine

- biliary tree

- stem cells

- organoids

Despite decades of basic research, biliary diseases remain prevalent, highly morbid, and notoriously difficult to treat. We have, however, dramatically increased our understanding of biliary developmental biology, cholangiocyte pathophysiology, and the endogenous mechanisms of biliary regeneration and repair. All of this complex and rapidly evolving knowledge coincides with an explosion of new technological advances in the area of regenerative medicine. New breakthroughs such as induced pluripotent stem cells and organoid culture are increasingly being applied to the biliary system; it is only a matter of time until new regenerative therapeutics for the cholangiopathies are unveiled. In this review, the authors integrate what is known about biliary development, regeneration, and repair, and link these conceptual advances to the technological breakthroughs that are collectively driving the emergence of a new global field in biliary regenerative medicine.
\end{abstract}

Because of the unique regenerative ability of the liver, many decades of research into liver regeneration have given us unique insights into various modes of organ regeneration ${ }^{1}$; therefore, hepatology has historically been at the epicenter of the science of regeneration. Although the underpinnings of modern regenerative medicine have been developing for much of that time, the worldwide pivot toward regenerative medicine, which now pervades all of modern medicine, can be most clearly landmarked by the Nobel Prize-winning technology of induced pluripotent stem cells, reported a decade ago in $2006 .^{2}$ Although this technology was quickly adapted to the study of hepatocyte differentiation, ${ }^{3-12}$ hepatocellular disease modeling, ${ }^{13-16}$ and cell-based therapy, ${ }^{17-19}$ there has been a subsequent expansion of similar advances in the realm of biliary disorders. ${ }^{20-23}$ As we will see, the biliary tree has always been center stage in the quest to understand the regenerative capacities and limitations of the liver because it has a distinct developmental origin, is anatomically and functionally heterogeneous, harbors several putative stem cell niches, and is the target for a vast array of liver pathologies. Furthermore, in many ways, the biliary tree may be an even more attractive target for regenerative therapeutics than parenchymal hepatocytes, given its broad distribution throughout the liver and its endoscopic accessibility by endoscopic retrograde cholangiopancreatography (ERCP). Recently, several excellent review articles have been published on individual aspects relevant to the topic of biliary regenerative medicine. ${ }^{24-27}$ However, a more comprehensive perspective has been lacking and may be useful to help further define this emerging field. In this review, we will revisit what is known about biliary development, regeneration, and repair; summarize current concepts related to biliary stem cells and cellular plasticity in the liver; review the current state of advances in biliary regenerative medicine; and provide a vision of where this field is most ideally poised for advances in basic discoveries and clinical applications in the future.

These authors contributed equally.

Copyright $\odot 2017$ by Thieme Medical Publishers, Inc., 333 Seventh Avenue, New York, NY 10001, USA.

Tel: +1(212) 584-4662.
DOI http://dx.doi.org/ $10.1055 / \mathrm{s}-0036-1597818$. ISSN 0272-8087. 


\section{Cholangiocytes and Cholangiopathies}

The liver, which is responsible for bile acid production, serum detoxification, the synthesis of serum proteins, immune regulation, and metabolic activities, is composed of two types of epithelial cells: hepatocytes and cholangiocytes. Although a majority of the essential functions are performed by hepatocytes, which make up 95\% of the liver parenchyma, specialized cholangiocytes form the biliary tree. The biliary tree is composed of intrahepatic and extrahepatic bile ducts, lined by the mature epithelial cholangiocytes. ${ }^{28}$ They facilitate secretion and modification of biliary constituents and serve as a conduit for bile transport to the intestine. These cells are now known to be the target of a diverse group of biliary disorders, known as cholangiopathies, many of which can lead to progressive periportal fibrosis, portal hypertension, biliary cirrhosis, and cholangiocarcinoma. ${ }^{29}$ The cholangiopathies can be proliferative (e.g., polycystic liver disease) or fibro-obliterative (e.g., primary sclerosing cholangitis) in character and have heterogeneous etiopathogenesis (e.g., genetic, toxic, immunomediated, vascular, etc.). ${ }^{30} \mathrm{~A}$ brief outline of five major cholangiopathies will demonstrate the breadth of clinical issues facing these patients.

Primary sclerosing cholangitis (PSC) is an idiopathic, fibroobliterative cholangiopathy characterized by the diffuse inflammation of intrahepatic and/or extrahepatic bile ducts. ${ }^{31}$ This chronic process can progress to end-stage biliary cirrhosis with portal hypertension and hepatic failure. Ursodeoxycholic acid (UDCA) has been used for the treatment of PSC and when administrated in low doses, has shown improvement in serum liver biochemistries. Vancomycin has also been used, particularly in children with PSC.

Primary biliary cholangitis (PBC) is a complex, autoimmunomediated cholangiopathy, characterized by the progressive destruction of the intrahepatic bile ducts, leading to cholestasis and portal inflammation, which when chronic, can progress to periportal fibrosis and cirrhosis. ${ }^{32}$ The prognosis for PBC has been improved with the use of UDCA. ${ }^{33}$ Recently, the Food and Drug Administration also approved obeticholic acid as a treatment for PBC.

Autosomal-dominant polycystic kidney disease is the most common inherited kidney disease, caused by mutations in the PKD1 and PKD2 genes. ${ }^{34}$ In many cases, autosomaldominant polycystic kidney disease is also associated with polycystic liver disease, a proliferative cholangiopathy associated with ciliary dysfunction in which multiple cysts develop within the liver parenchyma as a result of alterations in calcium homeostasis and cyclic adenosine monophosphate activity and subsequent effects on protein kinase-mediated proliferation.

Biliary atresia is a rare childhood disease that affects the function of and the anatomy along the canalicular-bile duct continuum. ${ }^{35}$ The obliteration or discontinuity of the extrahepatic biliary system results in the obstruction of bile flow, leading to cholestatic jaundice. Biliary atresia is treated with the Kasai hepatoportoenterostomy and/or liver transplantation. The condition remains the most common childhood indication for liver transplantation.
Cholangiocarcinoma is a group of rare, but devastating, hepatobiliary cancers that arise from the intrahepatic, perihilar, or distal biliary tree. ${ }^{36}$ Chronic inflammation from liver fluke infestation, hepatitis B and C infections, and PSC are the main risk factors for cholangiocarcinoma. Other etiologic factors include inflammatory bowel disease, hepatolithiasis, cirrhosis, alcohol, smoking, and fatty liver disease. A highly selected subgroup of patients with hilar cholangiocarcinoma can benefit from neoadjuvent chemoradiation followed by liver transplantation.

Overall, treatment of the cholangiopathies consists of pharmacotherapies (e.g., UDCA, vancomycin, etc.) that are largely ineffective and surgical therapies (e.g., Kasai procedure, liver transplantation, etc.) that are limited in scope and availability. ${ }^{37}$ As such, many times cholangiopathies remain essentially untreatable with high morbidity and mortality in both children and adults. Even after successful liver transplantation, patients can be stricken with devastating biliary complications such as ischemic cholangiopathy (in the case of donation after cardiac death or hepatic artery thrombosis). Living donor transplant (effectively a regenerative medicine therapy) has significantly expanded the pool of donor organs, but unfortunately, biliary complications such as strictures and leaks are notoriously prevalent following this procedure, effecting up to $20 \%$ of living donor recipients.

\section{Biliary Regenerative Medicine and the $\mathbf{R}^{3}$ Paradigm}

The unfortunate lack of effective therapies for biliary disease has prompted the aggressive evaluation of new therapeutic options in the realm of biliary regenerative medicine that have the potential to radically alter our management of these patients. An expanding understanding of biliary pathophysiology, the presence of endogenous stem cell niches within the biliary tree, and endoscopic access to the system make cholangiopathies attractive targets for regenerative medicine therapies. At the most simplistic level, biliary regenerative medicine is tasked with creating new cholangiocytes and building new bile ducts. Although there is no definitive blueprint for the development of regenerative medicine therapies, one useful concept is the $\mathrm{R}^{3}$ paradigm, which incorporates three distinct regenerative tactics: replacement, regeneration, and rejuvenation. ${ }^{38}$ Replacement strategy refers to the transplantation of a cell-based product that reestablishes homeostasis (liver transplantation is an example of this approach). ${ }^{39,40}$ Regenerative strategy refers to the engraftment of progenitor cells that require in vivo growth and differentiation (stem cell transplant is an example). ${ }^{17,41,42}$ Rejuvenation strategy refers to the induction of self-renewal of tissues by the activation of endogenous stem cells. $^{43-45}$ In the context of biliary disease, replacement would include therapies designed to directly replace the damaged biliary epithelium (e.g., cholangiocyte-based cell therapies, bioengineered tissue patches, etc.). Regeneration, in contrast, would encompass stem cell-based therapies (biodegradable stem cell-coated stents, for example). Lastly, rejuvenation therapies would be designed to activate a 


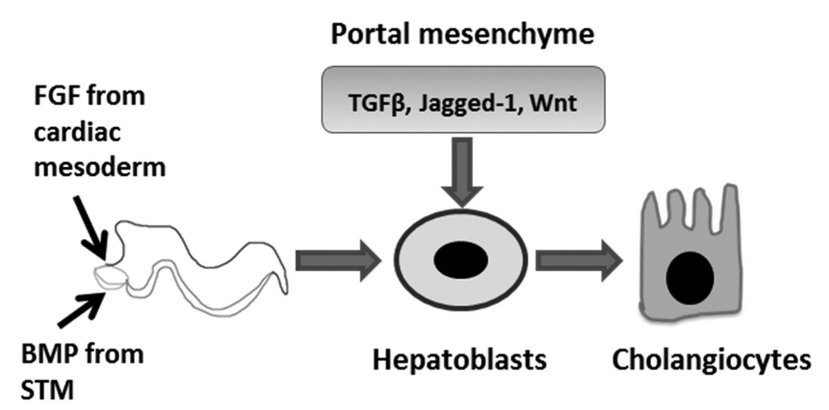

Fig. 1 Spatiotemporal regulation of cholangiocyte development. Schematic representation of key factors involved in biliary development from hepatoblasts. FGF, fibroblast growth factor; BMP, bone morphogenetic protein; STM, septum transversum mesenchyme; TGF $\beta$, transforming growth factor $\beta$.

therapeutic subset of the endogenous biliary stem/progenitor cell systems (gene therapy, therapeutic exosome delivery, etc.).

\section{Biliary Development}

To properly envisage new regenerative therapeutics for biliary disorders, it is useful, if not mandatory, to understand the normal embryological development of the biliary tree. The liver is formed from the ventral foregut endoderm, which also gives rise to the lung, the ventral pancreas, and the thyroid. ${ }^{46}$ The transcription of liver specific genes, such as albumin, can be detected in the ventral foregut endoderm as early as embryonic day 8.5 (E8.5), specifying hepatic differentiation. ${ }^{47}$ This hepatic induction is dependent on distinct, spatiotemporal regulation including signals of fibroblast growth factor (FGF) and bone morphogenetic protein (BMP) from the cardiac mesoderm and the septum transversum mesenchyme, respectively (- Fig. 1). ${ }^{47-50}$ Subsequent to the FGF and BMP signaling cascades, Wnt signaling from the mesoderm is also required for liver specification. ${ }^{51,52}$

Between E9.0 and E9.5, hepatic endoderm cells called hepatoblasts delaminate from the epithelium and expand into the adjacent septum transversum mesenchyme to form the liver bud, coordinated by signals from endothelial cells and a series of transcriptional events. ${ }^{53-55}$ Sonic hedgehog ( $\mathrm{SHH})$ is expressed in the ventral foregut endoderm during development, but at the onset of liver bud formation, its expression is downregulated. At E11.5, hepatoblasts show expression of $\mathrm{SHH}$ and its downstream transcription factor, Gli-1, which are then later attenuated. Thus, a temporally restricted activation of $\mathrm{Hh}$ signaling appears to be required to promote hepatoblast proliferation, a signal that is then shut off for normal hepatic differentiation of the hepatoblasts. ${ }^{56}$

The hepatoblasts are bipotent and differentiate into both cholangiocytes and hepatocytes beginning around E13. Liver bud hepatoblasts residing adjacent to the portal tracts, upon the influence of the portal mesenchyme, adopt a cholangiocyte fate and form the lumen of the intrahepatic bile ducts, while the hepatoblasts in the parenchyma continue to differentiate toward hepatocytes. The maturation and specification of these cells are regulated by diverse growth factors, cytokines, and transcription factors, which have been reviewed in detail elsewhere. ${ }^{24,57}$

Parenchymal hepatocyte differentiation requires exposure to oncostatin M secreted from the hematopoietic cells in the liver in combination with hepatocyte growth factor and Wnt hormones. ${ }^{58,59}$ The activity of these factors is further balanced by tumor necrosis factor $\alpha$, which maintains the proliferation of fetal hepatocytes for appropriate liver growth. These signals together regulate a network of liver-enriched transcription factors that control hepatocyte gene expression.

The biliary fate of the periportal hepatoblasts is orchestrated through temporally coordinated transforming growth factor (TGF) $\beta$, Notch, Wnt, and FGF signaling (-Fig. 1).$^{60-65}$ Jagged-1 (Jag-1), a Notch ligand, is a key signaling molecule for biliary development, and is thought to be derived from the portal mesenchyme. Deletion of the Jag- 1 gene in the portal mesenchyme results in profound defects in bile duct formation. ${ }^{66}$ In humans, mutations in Jag- 1 or Notch2 lead to bile duct paucity in patients with Alagille's syndrome. ${ }^{67-70}$ Furthermore, biliary differentiation is prevented by inhibiting Notch signaling, whereas ectopic Notch signaling promotes parenchymal hepatoblasts to adopt a biliary fate. ${ }^{62-64}$ Signaling through the Jag-1/Notch2 ligand-receptor pair, essential for biliary morphogenesis, is evolutionarily conserved in vertebrate liver development. ${ }^{66,71,72}$

Another important signaling pathway essential for biliary development is the TGF $\beta$ /activin pathway. A gradient of TGF $\beta$ signaling exists in fetal liver, with high levels in the periportal region and low levels in the pericentral region, which controls the induction of the biliary fate. Wnt signals also regulate biliary differentiation from the hepatoblasts. ${ }^{73}$ Taken together, a combination of spatially restricted signaling factors collectively allow for biliary differentiation in the periportal region.

\section{Biliary Regeneration and Repair}

Among the solid organs, liver is distinguished in its unique and remarkable capacity to regenerate upon injury (surgical resection or toxic insults). Humans can tolerate a $70 \%$ hepatectomy, rodents can tolerate a $90 \%$ hepatectomy, and zebrafish liver can regenerate after near total obliteration of parenchymal hepatocytes. ${ }^{74,75}$ Although the bulk of historical work on liver regeneration focuses on hepatocyte regeneration, restoration of the biliary tree is also essential for proper organ function. Additionally, the periportal location of putative liver stem cell niches and the expansion of biliary progenitors seen in the context of chronic liver diseases puts cholangiocytes at center stage in discussions of liver regeneration and repair mechanisms. ${ }^{25}$ In contrast to biliary development, which occurs through the differentiation of hepatoblasts, it is useful to classify biliary regeneration and repair into three distinct domains: (1) homeostatic selfreplication of cholangiocytes in normal liver, (2) accelerated biliary regeneration after liver resection, and (3) repair of the biliary tree upon injury (-Fig. 2 ).

One of the early proposed models for liver cell replacement was the so-called streaming liver hypothesis, where a 


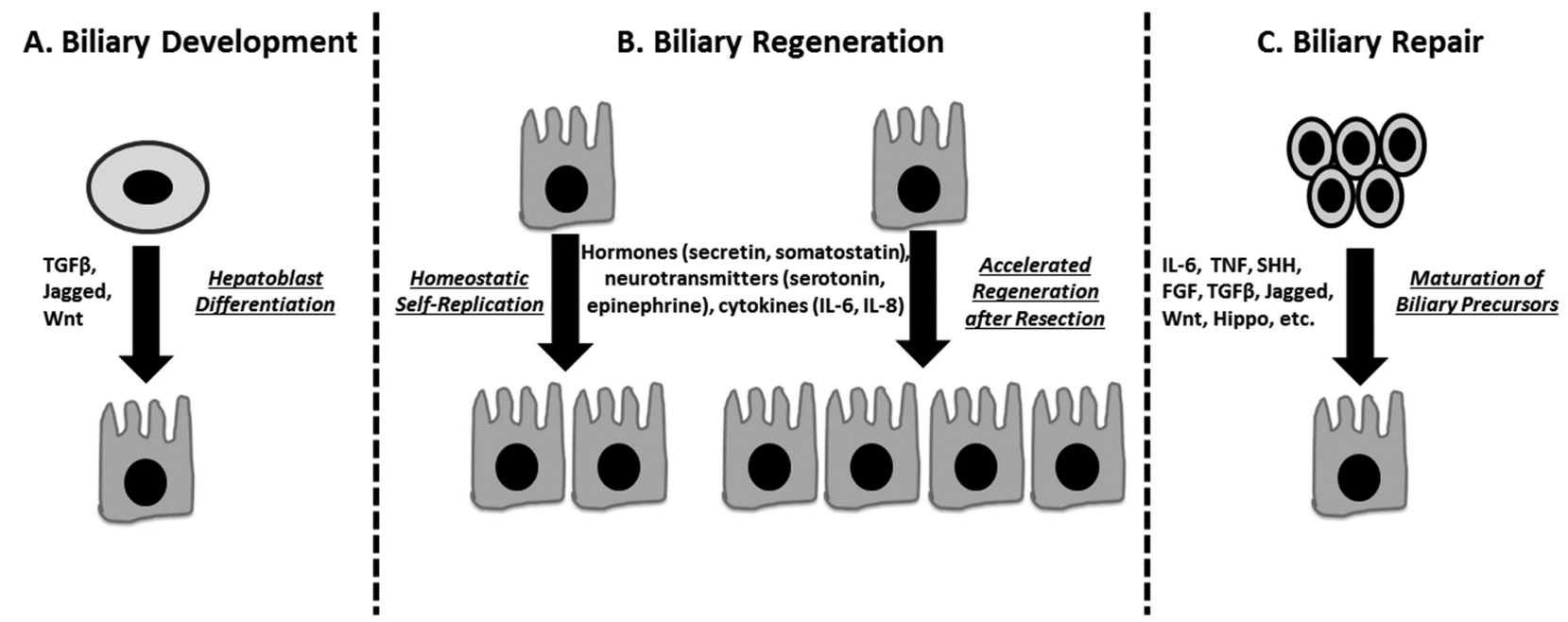

Fig. 2 Biliary development, regeneration, and repair. (A) Biliary development involving differentiation of hepatoblasts. (B) Biliary regeneration involving homeostatic self-replication of cholangiocytes in normal liver or accelerated self-replication following partial hepatectomy. (C) Biliary repair involving activation and maturation of biliary precursors.

continuous flow of new hepatocytes would emerge from the periportal stem cell niche and progress toward the central vein region for eventual apoptosis, analogous to the crypt-tovilli flow of intestinal epithelial cells. ${ }^{76}$ Although elegant, lineage-tracing evidence eventually accumulated and argued against such a model ${ }^{77,78}$ and it is now fairly well-established that normal liver tissue maintenance is achieved via homeostatic self-replication of pre-existing hepatocytes and cholangiocytes. Occasional and apparently random apoptosis of adult cells is counterbalanced by occasional and apparently random mitotic events.

The partial hepatectomy $(\mathrm{PHx})$ model in rat was the first classical model of liver regeneration, described by Higgins and Anderson in $1931 .^{79}$ They surgically excised two anterior lobes of rat liver, equating to $70 \%$ reduction in liver size. The cells of the remaining lobes proliferated to regain the lost mass over the course of 1 to 2 weeks. In this process, liver cells are able to overcome cell-cycle checkpoints and re-enter the cell cycle. This is followed by waves of DNA synthesis, cellular hypertrophy, and proliferation. Organ mass and function is eventually restored through compensatory hypertrophy of the remnant lobes as opposed to regrowth of resected lobes. There is apparently little activation of the periportal stem cell niche in this circumstance, arguing that normal liver regeneration can be achieved by simple proliferation of adult parenchymal cells without invoking any stem/progenitor cell population.

However, when the liver suffers from severe and/or chronic damage, cellular proliferation and regenerative capacity are thought to be attenuated. In this circumstance, historical paradigms have suggested that there is emergence of a facultative bipotent liver stem/progenitor cell compartment to contribute to the process of liver regeneration. These stem/ progenitor cells are also referred to as oval cells in rodent models due to their stem cell-like morphology, ${ }^{80,81}$ and have been extensively evaluated as a potential source of new hepatocytes during liver regeneration. ${ }^{25,82}$ Even with sup- pressed hepatocyte proliferation in rats by the administration of the chemical acetylaminofluorene, followed by PHx, oval cells can appear and expand in the periportal regions of the lobule within a few days and reach a peak at $\sim 7$ to 9 days after PHx. The administration of certain diets such as 3,5-diethoxycarbonyl-1,4-dihidro-collidine ${ }^{83}$ feeding or the choline-deficient, ethanolamine supplemented ${ }^{84}$ diet are well-known to produce robust oval cell responses in both mice and rats. Cells bearing a strong resemblance to rodent oval cells are also observed in human liver diseases. In humans, however, these cells are usually termed "hepatic progenitor cells" or "intermediate hepatobiliary cells. ${ }^{85}$ The hematopoietic and epidermal systems as well as the small intestine have defined stem cell populations responsible for normal cell turnover that have been isolated and anatomically localized. These stem cells exhibit self-renewal properties and differentiate into mature cell types under normal physiological conditions to replace cell losses in blood, skin, and gut, thereby maintaining normal tissue homeostasis. In the liver, normal hepatocyte and cholangiocyte turnover is slow, and the concept of such a stem cell niche to support homeostatic self-renewal or tissue repair after injury has long been contemplated. The lack of a well-organized and universally accepted system of nomenclature for ductular cells and biliary precursors has led to a proliferation of poorly understood and loosely applied terminology (e.g., liver progenitor cells, biliary tree stem cells, atypical ductular cells, oval cells, biliary tree stem/progenitors, ductular hepatocytes, intermediate hepatocytes, reactive cholangiocytes). Although each term is meant to strictly refer to a specific subpopulation of a heterogeneous group of cells along the biliary lineage, the terms are often used synonymously and interchangeably, which has led to some confusion in the literature. Perhaps the simplest term to apply to the diverse expansion of the biliary compartment seen in chronic liver disease is the descriptive term, "ductular reactive cells" (DRCs), which describes the emergence of a histological lesion, known as the ductular reaction. The ductular 
reaction consists of the activation and expansion of immature cholangiocytic cells that coalesce into primitive duct-like structures. The ductular reaction is, in fact, a collective signaling response to the surrounding hepatic stellate cells, macrophages, mature cholangiocytes, hepatocytes, portal myofibrobalsts, and endothelial cells, which form a niche that regulates the formation, expansion, and differentiation of DRCs. ${ }^{86}$ This reaction is apparently an attempt to activate endogenous repair mechanisms, but can also be viewed as an abnormal regenerative response because it is accompanied by excessive extracellular matrix deposition and promotes the progression of fibrosis. ${ }^{87-89}$

The origin and fate of the ductular reactive cells is a subject of active debate in the literature and a topic on which concepts are rapidly evolving. What is clear is that a subpopulation of immature biliary-like cells is highly expanded in certain forms of chronic liver injury and that these cells have a bipotent differentiation capacity when isolated in vitro. There are varying theories on the origin of DRCs including a purely biliary origin, or alternatively, transdifferentiation of mature hepatocytes. Once established, however, the ductular reaction appears to be composed of cells of a biliary phenotype based on the following: (1) Many known oval cell markers are also markers for cholangiocytes ${ }^{90,91}$; (2) the arrangement of these cells histologically is often in a ductular pattern ${ }^{92}$; and (3) they typically emanate from and cluster near the portal tracts. ${ }^{93}$ Historically, the canals of Hering, terminal structures where the hepatocyte canaliculi and the interlobular bile ducts interconnect, have been proposed as the site of origin for DRCs. Given the ideal anatomical location of this structure between the two epithelial cells, it was reasonable to postulate that it could serve as a niche to supply the putative stem cells for both the hepatocytes and cholangiocytes. Direct proof of this model is hampered due to a lack of specific marker proteins and the inexact nature of lineage-tracing studies. Additional stem cell niches are being described including the peribiliary glands and a maturational lineage of stem and progenitor cells along the length of the biliary tree. $^{94}$ Recent studies also suggest that a self-renewing population of Axin2+ hepatocytes can regenerate damaged liver from a pericentral location through a reverse-streaming mechanism. ${ }^{95}$

\section{Stem Cells and Cellular Plasticity in Liver}

In addition to the complexities surrounding the origin of DRCs, an even more relevant question is their ultimate fate ( $i$. e., do ductular progenitors give rise to mature cholangiocytes to facilitate biliary repair and/or do they also contribute to parenchymal hepatocyte regeneration?). Pluripotent stem cell technology has revealed that adult cells, previously thought to be "terminally differentiated" retain remarkable cellular plasticity. This seems to be particularly true in the liver where complex transdifferentiation events are being documented, raising questions about whether cellular plasticity and reprogramming events may obviate the need for stem cell-based liver regeneration. ${ }^{96-98}$ The idea of biliaryderived DRCs being bipotent in vivo has been supported by lineage-tracing studies utilizing Cre/Lox technology with various Cre drivers and injury models; however, there are also several studies that do not support or show minimal contribution of biliary cells to regenerating hepatocytes (-Table 1). ${ }^{77,78,99-107}$ Studies done in zebrafish suggest that biliary cells contribute to hepatocyte restoration only in severe but not in moderate hepatocyte ablation. ${ }^{74,75}$ Another recent study in mice also showed that following deletion of $98 \%$ of hepatocytes, transplanted cells of biliary origin contributed significantly to the restoration of liver parenchyma, regenerating both hepatocytes and cholangiocytes. ${ }^{106}$ The key limitations in definitively resolving the apparent contradictory findings lie in the imperfect and variable models of liver disease available (none of which

Table 1 Lineage tracing studies utilizing various Cre drivers and injury models to study the fate of ductular reactive cells

\begin{tabular}{|l|l|l|}
\hline Reference & Genetic mice for lineage tracing & Injury model \\
\hline Sackett et al, $2009^{99}$ & Foxl1-Cre & BDL, DDC, CDE \\
\hline Furuyama et al, $2011^{100}$ & Sox9 Cre & CCl4, PHx, CDE, BDL, DDC, APAP \\
\hline Malato et al, $2011^{77}$ & Ttr-Cre & CCl4, PHx, BDL, DDC \\
\hline Espanol-Suner et al, $2012^{78}$ & OPN-Cre & PHx, CCl4, CDE, DDC \\
\hline Huch et al, $2013^{101}$ & LGR5-Cre & CCl4, MCDE, DDC \\
\hline Rodrigo-Torres et al, $2014^{102}$ & HNF1ß-Cre & CCl4, PHx, CDE, DDC, APAP \\
\hline Schaub et al, $2014^{103}$ & Ttr-Cre, CK19-Cre, PDGFR- 3 -Cre & CDE \\
\hline Tarlow et al, $2014^{104}$ & Sox9-Cre & PHx, CCl4, CDE, DDC \\
\hline Yanger et al, $2014^{105}$ & KRT-19-Cre, TBG-Cre & CCl4, CDE, DDC, ANIT \\
\hline Lu et al, $2015^{106}$ & KRT-19-Cre & CDE, $\Delta M d m 2$ \\
\hline Kamimoto et al, $2016^{107}$ & Prom1-Cre, AAV8-iCre & TAA, DDC \\
\hline
\end{tabular}

Abbreviations: AAV8, adeno-associated virus 8; ANIT, alpha napthylisothiocyanate; APAP, acute acetaminophen; BDL, bile-duct ligation; CCl4, carbon tetrachloride; CDE, choline-deficient ethionine supplemented; DDC, 3,5-diethoxycarbonyl-1,4-dihiro-collidine; Foxl1, forkhead box I1; HNF1 $\beta$, hepatocyte nuclear factor 1 homeobox B; KRT19/CK19, cytokeratin 19; LGR5, leucine-rich repeat-containing G-protein coupled receptor 5; OPN, osteopontin; PHx, partial hepatectomy; Prom-1, prominin-1; Sox9, SRY-related HMG-box transcription factor 9; TAA, thioacetamide; TBG, thyroid hormone-binding globulin; Ttr, transthyretin. 
accurately represent human chronic liver disease) and some technical limitations of lineage-tracing studies. Genetic lineage tracing is a powerful strategy for in vivo fate-tracing experiments because it allows for cell-type specificity (using cell-specific Cre drivers) and Cre expression can be controlled temporally (with tamoxifen-inducible systems). Although lineage tracing remains the gold standard to trace the origin of new cells, several important caveats need to be kept in mind. ${ }^{108,109}$ Leaky or unexpected Cre expression in different cell types, even in small amounts, can bias results. Injury states may alter the cell-specificity of Cre. Furthermore, tamoxifen can persist within the animal so that temporal precision of labeling is not always possible. Tamoxifen is also excreted in feces and can lead to Cre activation in untreated animals if co-housed. Despite these known limitations, genetic lineage tracing is likely to continue to provide novel and important insights into liver cell plasticity in the future.

\section{Stem Cell-Derived Cholangiocytes}

Human embryonic stem cells and induced pluripotent stem cells (iPSCs) have self-renewal capacity, are pluripotent, and have the ability to differentiate into cells of all three primary germ layers. Soon after the development of iPSCs, several in vitro hepatocyte differentiation strategies quickly emerged. Although these protocols produced cells with many features of adult hepatocytes, it has been notoriously difficult to achieve a fully mature adult hepatocyte phenotype; because of this, the differentiated cells have typically been referred to as hepatocyte-like cells. Nonetheless, these protocols provide powerful tools for studying liver developmental biology, recapitulating specific disease phenotypes, and could potentially provide unlimited resources for drug-testing applications and cell-based therapies.

The natural and logical evolution of the application of iPSC technology to liver disease was to develop iPSC-derived cholangiocytes for modeling biliary disease. Indeed, several groups have now published unique protocols for the differentiation of iPSCs into cholangiocytes. The protocols available for the differentiation of stem cells to cholangiocytes were mainly designed to mimic the patterns and stages observed during biliary development in utero. All were designed with a stepwise approach in which the cells were exposed to soluble factors as they proceed through various phases of endodermal differentiation. In principle, these phases should mimic normal embryologic development through phases including a definitive endoderm phase, a hepatic specification phase, a hepatoblast-like phase, and a differentiated cholangiocyte phase (-Table 2).

In 2014, Dianat et al showed that embryonic stem cells could be differentiated to cholangiocytes and subsequently applied the technique to iPSCs and HepaRG cells. They generated hepatoblasts using methods previously described in the generation of hepatocytes from pluripotent cells. In a monolayer, cells in the hepatoblast stage were matured to cholangiocytes with exposure to human growth hormone, epidermal growth factor (EGF), interleukin 6 (IL6 ), and sodium taurocholate. Cells from the final stage of differentiation expressed high levels of cholangiocyte markers such as CK7, CFTR, TGR5, HNF6, SOX9, and AQP1. The cells also formed cilia and when cultured in a threedimensional (3D) matrix, they developed epithelial/apicobasal polarity, and they formed functional cysts and biliary ducts. ${ }^{20}$

Our group utilized defined media and feeder-free culture conditions along with temporal exposure to key biliary morphogens to achieve cholangiocyte differentiation from patient-derived iPSCs. We reported the use of temporally restricted Hh signaling during the differentiation of iPSC to cholangiocytes. Hepatic specification was achieved using a combination of SHH, BMP4, and FGF2. To induce hepatic progenitor cells, SHH was also used in combination with Jag-1 (to activate Notch signaling). For the cholangiocyte maturation, TGF $\beta$ was used in conjunction with a collagen1 matrix. The resulting cholangiocytes showed expression of cholangiocyte markers (CK19, CK7, PKD2, CFTR, AE2), the presence of primary cilia, intact calcium signaling, and were able to form duct-like structures in 3D culture. This protocol also showed that iPSC-derived cholangiocytes were able to engraft within mouse liver in vivo, following retrograde intrabiliary infusion. ${ }^{21}$

Table 2 Growth factors employed by different groups for differentiation of stem cells to biliary cells

\begin{tabular}{|l|l|l|l|l|}
\hline Reference & Definitive endoderm & Hepatic specification & Hepatic progenitor & Cholangiocyte \\
\hline $\begin{array}{l}\text { Dianat et al, } \\
2014^{20}\end{array}$ & Wnt3A, ActivinA, FGF2 & ActivinA, FGF2, BMP4 & FGF4, HGF, EGF, RA & HG, EGF, IL-6, ST \\
\hline De Assuncao et al, 201521 & Wnt3A, ActivinA & SHH, BMP4, FGF2 & Jagged1, SHH & TGF $\beta$ \\
\hline $\begin{array}{l}\text { Ogawa et al, } \\
2012^{22}\end{array}$ & ActivinA & FGF2, BMP4 & HGF, Dex, OSM & $\begin{array}{l}\text { HGF, EGF, TGF } 3 \text {, } \\
\text { OP9 co-culture }\end{array}$ \\
\hline $\begin{array}{l}\text { Sampaziotis et al, } \\
2015^{23}\end{array}$ & ActivinA,FGF2, BMP4 & BMP4 & FGF10, ActivinA, RA & EGF \\
\hline $\begin{array}{l}\text { Takayama et al, } \\
2016^{110}\end{array}$ & ActivinA & FGF4, BMP4 & Dex, HGF, EGF & EGF, IL-6, ST \\
\hline
\end{tabular}

Abbreviations: BMP, bone morphogenetic protein; Dex, dexamethasone; EGF, epidermal growth factor; FGF, fibroblast growth factor; HG, human growth hormone; HGF, hepatocyte growth factor; IL-6, interleukin-6; OSM, oncostatin M; RA, retinoic acid; SHH, sonic hedgehog; ST, sodium taurocholate; TGF $\beta$, transforming growth factor $\beta$. 
Ogawa et al used a combination of hepatocyte growth factor (HGF), dexamethasone, and oncostatin to obtain hepatic progenitors and a subsequent combination of HGF, EGF, and TGF $\beta$ to obtain mature cholangiocytes. Notch signaling was activated using a co-culture with OP9 cells, a stromal cell line that expresses Notch ligands. The resulting cells, when grown in 3D culture, formed epithelialized cystic and/or ductular structures that expressed markers found in mature cholangiocytes. This study also showed that cholangiocytes generated from the iPSCs of patients with cystic fibrosis had impaired cyst swelling and that CFTR chemical correctors increased the levels of CFTR on the apical side of the lumen and augmented cyst swelling. ${ }^{22}$

Sampaziotis et al showed that in their protocol FGF10, retinoic acid, and TGF $\beta$ were key factors for biliary specification. Their iPSC-derived cholangiocytes were characterized using a range of assays that resembled primary common bile duct cholangiocytes at the transcriptional level. Patient-derived iPSCs in 3D culture conditions were used to model polycystic liver disease and cystic fibrosis. ${ }^{23} 3 \mathrm{D}$ cultures of iPSC-derived cholangiocytes generated from polycystic patients were responsive to secretin and somatostatin; this response was blunted by the use of octreotide. They also showed that the cholangiocytes derived from cystic fibrosis patients had minimal CFTR protein expression and were unable to modify intracellular chloride. They corrected the disease phenotype with an experimental drug, VX809.

Takayama et al used an approach similar to the Dianat protocol to promote cholangiocyte differentiation. The maturation of their hepatoblast-like cells was done with human growth hormone, EGF, and IL-6 along with extracellular matrix molecules. The gene-expression levels of the cholangiocyte markers, AQP1, SOX9, CFTR, G protein-coupled bile acid receptor 1 , Jag-1, secretin receptor, and GGT were all increased by using laminin 411 or laminin 511 as a matrix. ${ }^{110}$

Overall, the collective efforts at generating cholangiocytes derived from stem cells have been remarkably successful. These cells are already proving to be promising tools that can be used in many applications such as cell transplantation studies, biliary disease modeling, deciphering biliary development, and small molecule screening. Although each of the various differentiation protocols have some similarities and some unique aspects, it is fascinating that the disparate protocols all seem to result in a mature, adult cholangiocyte phenotype, insofar as these features have been evaluated. This may suggest that in vitro the default differentiation pathway of liver progenitors is biased toward cholangiocyte differentiation, especially given the difficulties noted in generating fully mature stem cell-derived hepatocytes. This is in contradistinction to the conceptualized sequence of events in liver development, where it is thought that intervening signals from the portal mesenchyme are required for cholangiocyte differentiation, whereas the "default" fate for most hepatoblasts is thought to be hepatocellular. Most likely, additional fine-tuning of the various cholangiocyte differentiation approaches will be needed to achieve the most robust and efficient cholangiocyte differentiation possible. It may also be that certain protocols will have advantages or disadvan- tages in certain applications. Regardless, this powerful new technology has provided unique opportunities to study cholangiocyte development, pathogenesis, and treatment strategies in ways that were previously unthinkable. When combined with large-scale efforts that are underway to generate robust biorepositories of iPSCs, iPSC-derived cholangiocytes, and biliary organoids from patients with cholangiopathies, this technology will become an even more powerful tool for individualized medical applications.

\section{Three-Dimensional Culture Systems and Biliary Organoids}

Organoids are collections of organ-specific cell types that develop from stem cells or organ progenitors. ${ }^{111}$ Organoid formation recapitulates the major processes of self-organization during development including cell sorting and spatially restricted lineage commitment in a manner similar to the in vivo environment. ${ }^{112}$ Grouped together and spatially organized similar to an organ, these structures are capable of recapitulating specific functions of the adult organs (e.g., secretion / absorption, filtration, neural activity, contraction, etc.). An important distinction to bear in mind is the difference between 3D cultures of a single-cell type and true organoids, which consist of multiple self-organizing cell types. An example of this distinction from the intestine is the difference between an enteroid (which consists of a single-cell type in 3D culture) and a true intestinal organoid (a stem cell-derived and self-organizing multicellular cluster). Organoid research has tremendous potential across multiple organ systems to facilitate disease modeling, pharmacological testing, and therapeutic regenerative medicine applications (-Fig. 3). Indeed, organoids have been generated to model

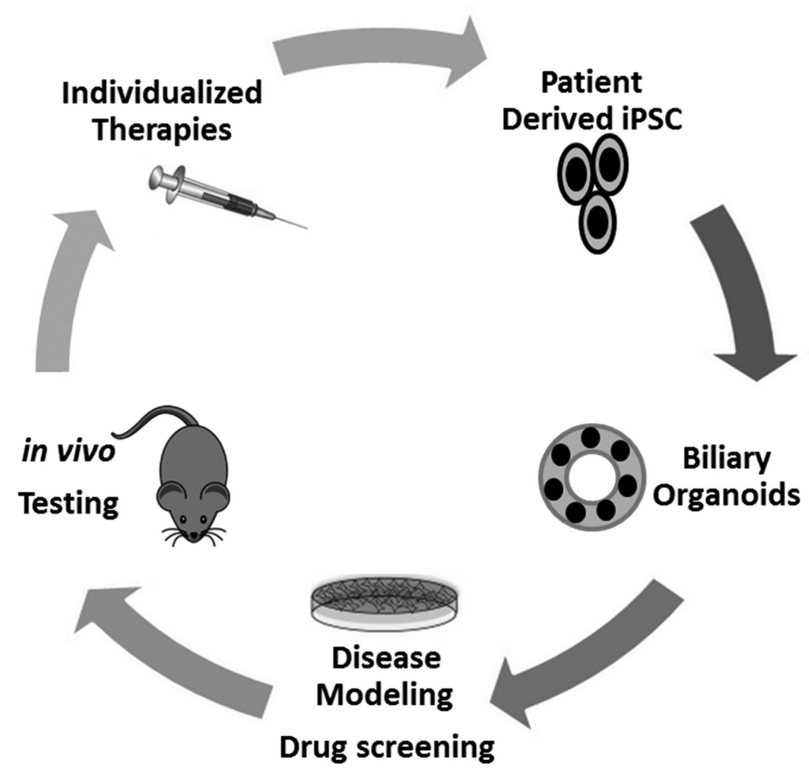

Fig. 3 Induced pluripotent stem cell- (iPSC-) derived cholangiocytes and applications. Schematic depicting patient-derived iPSC that could potentially be expanded as organoids, utilized for disease modeling and drug screening, tested in vivo, and eventually returned to the patient as an individualized therapy. 
diverse organs including the kidney, the lung, the brain, the thyroid, and others. ${ }^{111}$ Within gastroenterology, organoid systems have been generated to represent the esophagus, the stomach, the pancreas, the small intestine, the large intestine, and the pancreas. ${ }^{113}$ Liver organoids have been generated from both tissue-derived progenitor cells ${ }^{101,114}$ and from iPSCs. ${ }^{115}$ Organoid research is a logical extension of the existing iPSC technology that has been leveraged toward the biliary system. Furthermore, the infrastructure for overseeing biorepositories of biliary organoids likely already exists within existing iPSC biorepositories.

Several groups have utilized 3D culture systems to facilitate research in primary liver stem cells. As early as 2001, it was shown that primary liver cells cultured under 3D conditions could be maintained in vitro in the presence of EGF, HGF, and dexamethasone. ${ }^{116}$ Mouse Foxl1 ${ }^{+}$hepatic progenitor cells, when cultured in type 1 collagen gels, formed CK19 positive branches. ${ }^{117}$ In 2007, Tanimizu et al showed that HPPL, a mouse liver progenitor cell line, when cultured in 3D, formed cysts with a luminal space and apicobasal polarity. ${ }^{118}$ Kido et al showed that $\mathrm{CPM}^{+}$cells, sorted and matured to cholangiocytes in 3D culture, formed cysts with a luminal structure and proper apicobasal polarity. ${ }^{119}$ Recently, it was also shown that rat liver stem cells can be isolated and grown as cystic structures when cultured in high levels of Wnt3a and noggin (a BMP signaling inhibitor). Gallbladder stem/progenitor cells from noninjured livers can form 3D structures that express stem cell markers in the presence of R-spondin 1 , noggin, and nicotinamide. ${ }^{120}$ Yu et al published a protocol for direct reprograming of fibroblasts to induced hepatic stem cells. These cells expressed hepatocyte and cholangiocyte markers in vitro and were able to form cysts and branching structures that were positive for CK19 and CK7 under 3D culture. $^{121}$

In 2013, an elegant study generated true liver organoids by combining human-iPSC-derived hepatic cells with mesenchymal stem cells and endothelial cells. Although the cells were cultured in two-dimensional conditions, the cells self-organized into 3D clusters, which resembled embryonic liver. ${ }^{115}$ When transplanted in mice, these organ buds developed into hepatic tissue with features of adult liver. Notably, however, these organoids lacked biliary elements. Huch et al have demonstrated that LGR5+ adult liver stem cells can be isolated and cultured as liver organoids in a 3D culture method involving matrigel with HGF, EGF, FGF, and Rspondin $1 .{ }^{101}$ The expanded cells self-organized into 3D structures with a ductal $\left(\mathrm{CK} 19^{+}\right)$single-layered epithelium compartment and a pseudo-stratified compartment expressing both ductal (CK7, CK19) and hepatocyte (E-cadherin, Hnf4 $\alpha$ ) markers. When cultured in 3D, a single LGR5 ${ }^{+}$cell can be expanded as organoids and differentiated into hepatocytes and cholangiocytes. In 2015, the same group used a similar approach to expand liver biopsy tissue and single $\mathrm{EPCAM}^{+}$ cells as liver organoids. ${ }^{114}$ As powerful as these emerging systems are, organoid technology does have some notable limitations. Because they are generated in vitro, organoids in culture may not fully recapitulate all aspects of liver development that occur in the in vivo environment. In particular, they lack several essential components of the intact liver such as a vascular system, interaction with other cell types, specific extracellular matrix interactions, and immune surveillance (although organoid transplantation studies may help to overcome some of these issues). Despite these known limitations, 3D culture systems and liver organoid technology are rapidly advancing and are likely to be increasingly utilized for individualized disease modeling and regenerative medicine applications for the biliary system.

\section{Summary}

In the past several decades, we have witnessed significant advances in our understanding of biliary development, the basic physiology of cholangiocytes, and the pathogenesis of the cholangiopathies. Despite these advances, effective treatment modalities remain elusive. For this reason, the recent explosion of work in biliary regenerative medicine is particularly encouraging and provides great hope for future regenerative therapeutics for biliary disease. In this review, we outlined some of the primary clinical challenges associated with the biliary system and we identified a standard regenerative medicine paradigm involving replacement, regeneration, and rejuvenation that may help to categorize the development of future regenerative therapies. We also highlighted both historical and more recent basic science advances in biliary development, regeneration, and repair, and we reviewed new regenerative technologies involving iPSC-derived cholangiocytes and biliary organoids. All of these conceptual and technical advances now set the stage for the future translation and application to ultimately develop new regenerative service lines for patients with biliary disease. When and how these new therapies will emerge is unknown, but their development and translation will likely require multidisciplinary transformational teams consisting of basic scientists, hepatologists, and biliary and transplant surgeons, as well as interventional radiologists and advanced endoscopists. It is likely that the field will take advantage of its existing endoscopic access to the biliary tree by building new platforms in regenerative endoscopy. The ERCP-based delivery of cell-based (e.g., stem cells or stem-cell derived cholangiocytes) or cell-free regenerative therapeutics (e.g., biodegradable stents or therapeutic exosome delivery) are particularly appealing because advanced endoscopy is available at academic centers throughout the world. Other technological applications being contemplated include 3D bioprinting of ductular tissue, biliary biostents incorporating cellular elements, and tissue-engineering approaches, such as recellularization of decellularized bile duct units. Our embrace of regenerative medicine also involves some challenges. We will need to face down the issues of inexact technologies, inefficient or incomplete differentiation from stem cells, epigenetic memory, and malignancy potential, as well as highly complex tissue engineering and regulatory challenges. In the end, however, we are left with great hope for clinical advances. Clearly, the future is bright in terms of regenerative medicine and the biliary tree. We are awakening to the dawn of a new golden age in regenerative hepatology that promises 
the development and application of new regenerative therapeutics for previously untreatable liver diseases.

\section{Financial Support}

This work was supported by grants DK100575, DK084567, and the Loan Repayment Program from the National Institutes of Health; by Regenerative Medicine Minnesota; by Gilead Sciences; and by the Mayo Clinic Center for Regenerative Medicine.

\section{Abbreviations}

$\begin{array}{ll}\text { 3D } & \text { three-dimensional } \\ \text { BMP } & \text { bone morphogenic protein } \\ \text { DRCs } & \text { ductular reactive cells } \\ \text { EGF } & \text { epidermal growth factor } \\ \text { ERCP } & \text { endoscopic retrograde cholangiopancreatography } \\ \text { FGF } & \text { fibroblast growth factor } \\ \text { HGF } & \text { hepatocyte growth factor } \\ \text { IL-6 } & \text { interleukin } 6 \\ \text { iPSCs } & \text { induced pluripotent stem cells } \\ \text { Jag-1 } & \text { Jagged-1 } \\ \text { PBC } & \text { primary biliary cholangitis } \\ \text { PHx } & \text { partial hepatectomy } \\ \text { PSC } & \text { primary sclerosing cholangitis } \\ \text { SHH } & \text { sonic hedgehog } \\ \text { TGF } & \text { transforming growth factor } \\ \text { UDCA } & \text { ursodeoxycholic acid }\end{array}$

\section{References}

1 Forbes SJ, Newsome PN. Liver regeneration - mechanisms and models to clinical application. Nat Rev Gastroenterol Hepatol 2016;13(8):473-485

2 Takahashi K, Yamanaka S. Induction of pluripotent stem cells from mouse embryonic and adult fibroblast cultures by defined factors. Cell 2006;126(4):663-676

3 Si-Tayeb K, Noto FK, Nagaoka M, et al. Highly efficient generation of human hepatocyte-like cells from induced pluripotent stem cells. Hepatology 2010;51(1):297-305

4 Sancho-Bru P, Roelandt P, Narain N, et al. Directed differentiation of murine-induced pluripotent stem cells to functional hepatocyte-like cells. J Hepatol 2011;54(1):98-107

5 Choi SM, Kim Y, Liu H, Chaudhari P, Ye Z, Jang YY. Liver engraftment potential of hepatic cells derived from patient-specific induced pluripotent stem cells. Cell Cycle 2011;10(15): 2423-2427

6 Chen YF, Tseng CY, Wang HW, Kuo HC, Yang VW, Lee OK. Rapid generation of mature hepatocyte-like cells from human induced pluripotent stem cells by an efficient three-step protocol. Hepatology 2012;55(4):1193-1203

7 Yu Y, Liu H, Ikeda Y, et al. Hepatocyte-like cells differentiated from human induced pluripotent stem cells: relevance to cellular therapies. Stem Cell Res (Amst) 2012;9(3):196-207

8 Aravalli RN, Cressman EN, Steer CJ. Hepatic differentiation of porcine induced pluripotent stem cells in vitro. Vet J 2012;194(3): 369-374

9 Zhu S, Rezvani M, Harbell J, et al. Mouse liver repopulation with hepatocytes generated from human fibroblasts. Nature 2014; 508(7494):93-97
10 Szkolnicka D, Farnworth SL, Lucendo-Villarin B, Hay DC. Deriving functional hepatocytes from pluripotent stem cells. Curr Protoc Stem Cell Biol 2014;30:1-12

11 Takayama K, Inamura M, Kawabata K, et al. Generation of metabolically functioning hepatocytes from human pluripotent stem cells by FOXA2 and HNF1 $\alpha$ transduction. J Hepatol 2012; 57(3):628-636

12 Schwartz RE, Fleming HE, Khetani SR, Bhatia SN. Pluripotent stem cell-derived hepatocyte-like cells. Biotechnol Adv 2014;32(2): 504-513

13 Cayo MA, Cai J, DeLaForest A, et al. JD induced pluripotent stem cell-derived hepatocytes faithfully recapitulate the pathophysiology of familial hypercholesterolemia. Hepatology 2012;56(6): 2163-2171

14 Xu D, Alipio Z, Fink LM, et al. Phenotypic correction of murine hemophilia A using an iPS cell-based therapy. Proc Natl Acad Sci U S A 2009;106(3):808-813

15 Yusa K, Rashid ST, Strick-Marchand H, et al. Targeted gene correction of $\alpha 1$-antitrypsin deficiency in induced pluripotent stem cells. Nature 2011;478(7369):391-394

16 Rashid ST, Corbineau S, Hannan N, et al. Modeling inherited metabolic disorders of the liver using human induced pluripotent stem cells. J Clin Invest 2010;120(9):3127-3136

17 Huebert RC, Rakela J. Cellular therapy for liver disease. Mayo Clin Proc 2014;89(3):414-424

18 Yu Y, Fisher JE, Lillegard JB, Rodysill B, Amiot B, Nyberg SL. Cell therapies for liver diseases. Liver Transpl 2012;18(1):9-21

19 Subba Rao M, Sasikala M, Nageshwar Reddy D. Thinking outside the liver: induced pluripotent stem cells for hepatic applications. World J Gastroenterol 2013;19(22):3385-3396

20 Dianat N, Dubois-Pot-Schneider H, Steichen C, et al. Generation of functional cholangiocyte-like cells from human pluripotent stem cells and HepaRG cells. Hepatology 2014;60(2):700-714

21 De Assuncao TM, Sun Y, Jalan-Sakrikar N, et al. Development and characterization of human-induced pluripotent stem cell-derived cholangiocytes. Lab Invest 2015;95(10):1218

22 Ogawa M, Ogawa S, Bear CE, et al. Directed differentiation of cholangiocytes from human pluripotent stem cells. Nat Biotechnol 2015;33(8):853-861

23 Sampaziotis F, Cardoso de Brito M, Madrigal P, et al. Cholangiocytes derived from human induced pluripotent stem cells for disease modeling and drug validation. Nat Biotechnol 2015; 33(8):845-852

24 Strazzabosco M, Fabris L. Development of the bile ducts: essentials for the clinical hepatologist. J Hepatol 2012;56(5):1159-1170

25 Itoh T. Stem/progenitor cells in liver regeneration. Hepatology 2016;64(2):663-668

26 Ghanekar A, Kamath BM. Cholangiocytes derived from induced pluripotent stem cells for disease modeling. Curr Opin Gastroenterol 2016;32(3):210-215

27 Hindley CJ, Cordero-Espinoza L, Huch M. Organoids from adult liver and pancreas: stem cell biology and biomedical utility. Dev Biol 2016;S0012-1606(16)30277-9

28 Tabibian JH, Masyuk AI, Masyuk TV, O'Hara SP, LaRusso NF. Physiology of cholangiocytes. Compr Physiol 2013;3(1):541-565

29 O'Hara SP, Tabibian JH, Splinter PL, LaRusso NF. The dynamic biliary epithelia: molecules, pathways, and disease. J Hepatol 2013;58(3):575-582

30 Lazaridis KN, Strazzabosco M, Larusso NF. The cholangiopathies: disorders of biliary epithelia. Gastroenterology 2004;127(5): 1565-1577

31 Halilbasic E, Fuchs C, Hofer H, Paumgartner G, Trauner M. Therapy of primary sclerosing cholangitis-today and tomorrow. Dig Dis 2015;33(Suppl 2):149-163

32 Carbone M, Ronca V, Bruno S, Invernizzi P, Mells GF. Toward precision medicine in primary biliary cholangitis. Dig Liver Dis 2016;48(8):843-850 
33 Mousa HS, Carbone M, Malinverno F, Ronca V, Gershwin ME, Invernizzi P. Novel therapeutics for primary biliary cholangitis: toward a disease-stage-based approach. Autoimmun Rev 2016; 15(9):870-876

34 Mikolajczyk AE, Te HS, Chapman AB. Gastrointestinal manifestations of autosomal-dominant polycystic kidney disease. Clin Gastroenterol Hepatol 2016:S1542-3565(16)30364-0

35 Verkade HJ, Bezerra JA, Davenport M, et al. Biliary atresia and other cholestatic childhood diseases: advances and future challenges. J Hepatol 2016;65(3):631-642

36 Rizvi S, Gores GJ. Pathogenesis, diagnosis, and management of cholangiocarcinoma. Gastroenterology 2013;145(6):1215-1229

37 Lazaridis KN, LaRusso NF. The cholangiopathies. Mayo Clin Proc 2015;90(6):791-800

38 Nelson TJ, Behfar A, Terzic A. Strategies for therapeutic repair: The " $R(3)$ " regenerative medicine paradigm. Clin Transl Sci 2008;1(2): $168-171$

39 Atala A. Advances in tissue and organ replacement. Curr Stem Cell Res Ther 2008;3(1):21-31

40 Mavila N, Trecartin A, Spurrier R, et al. Functional human and murine tissue-engineered liver is generated from adult stem/ progenitor cells. Stem Cells Transl Med 2016; doi: 10.5966/ sctm.2016-0205

41 Kørbling M, Estrov Z. Adult stem cells for tissue repair - a new therapeutic concept? N Engl J Med 2003;349(6):570-582

42 Nicolas CT, Wang Y, Nyberg SL. Cell therapy in chronic liver disease. Curr Opin Gastroenterol 2016;32(3):189-194

43 Surani MA, McLaren A. Stem cells: a new route to rejuvenation. Nature 2006;443(7109):284-285

44 Basu J, Ludlow JW. Exosomes for repair, regeneration and rejuvenation. Expert Opin Biol Ther 2016;16(4):489-506

45 Conboy IM, Conboy MJ, Rebo J. Systemic problems: a perspective on stem cell aging and rejuvenation. Aging (Albany, NY) 2015; 7(10):754-765

46 Tremblay KD, Zaret KS. Distinct populations of endoderm cells converge to generate the embryonic liver bud and ventral foregut tissues. Dev Biol 2005;280(1):87-99

47 Gualdi R, Bossard P, Zheng M, Hamada Y, Coleman JR, Zaret KS. Hepatic specification of the gut endoderm in vitro: cell signaling and transcriptional control. Genes Dev 1996;10(13): $1670-1682$

48 Jung J, Zheng M, Goldfarb M, Zaret KS. Initiation of mammalian liver development from endoderm by fibroblast growth factors. Science 1999;284(5422):1998-2003

49 Rossi JM, Dunn NR, Hogan BL, Zaret KS. Distinct mesodermal signals, including BMPs from the septum transversum mesenchyme, are required in combination for hepatogenesis from the endoderm. Genes Dev 2001;15(15):1998-2009

50 Serls AE, Doherty S, Parvatiyar P, Wells JM, Deutsch GH. Different thresholds of fibroblast growth factors pattern the ventral foregut into liver and lung. Development 2005;132(1):35-47

51 Goessling W, North TE, Lord AM, et al. APC mutant zebrafish uncover a changing temporal requirement for wnt signaling in liver development. Dev Biol 2008;320(1):161-174

52 Ober EA, Verkade H, Field HA, Stainier DY. Mesodermal Wnt2b signalling positively regulates liver specification. Nature 2006; 442(7103):688-691

53 Bort R, Signore M, Tremblay K, Martinez Barbera JP, Zaret KS. Hex homeobox gene controls the transition of the endoderm to a pseudostratified, cell emergent epithelium for liver bud development. Dev Biol 2006;290(1):44-56

54 Margagliotti S, Clotman F, Pierreux CE, et al. The onecut transcription factors HNF-6/OC-1 and OC-2 regulate early liver expansion by controlling hepatoblast migration. Dev Biol 2007; 311(2):579-589

55 Sosa-Pineda B, Wigle JT, Oliver G. Hepatocyte migration during liver development requires Prox1. Nat Genet 2000;25(3): 254-255
56 Hirose Y, Itoh T, Miyajima A. Hedgehog signal activation coordinates proliferation and differentiation of fetal liver progenitor cells. Exp Cell Res 2009;315(15):2648-2657

57 Zong Y, Stanger BZ. Molecular mechanisms of liver and bile duct development. Wiley Interdiscip Rev Dev Biol 2012;1(5):643-655

58 Kamiya A, Gonzalez FJ. TNF-alpha regulates mouse fetal hepatic maturation induced by oncostatin $\mathrm{M}$ and extracellular matrices. Hepatology 2004;40(3):527-536

59 Schmidt C, Bladt F, Goedecke S, et al. Scatter factor/hepatocyte growth factor is essential for liver development. Nature 1995; 373(6516):699-702

60 Decaens T, Godard C, de Reyniès A, et al. Stabilization of betacatenin affects mouse embryonic liver growth and hepatoblast fate. Hepatology 2008;47(1):247-258

61 Clotman F, Jacquemin P, Plumb-Rudewiez N, et al. Control of liver cell fate decision by a gradient of TGF beta signaling modulated by onecut transcription factors. Genes Dev 2005;19(16):1849-1854

62 Zong Y, Panikkar A, Xu J, et al. Notch signaling controls liver development by regulating biliary differentiation. Development 2009;136(10):1727-1739

63 Tchorz JS, Kinter J, Müller M, Tornillo L, Heim MH, Bettler B. Notch2 signaling promotes biliary epithelial cell fate specification and tubulogenesis during bile duct development in mice. Hepatology 2009;50(3):871-879

64 Tanimizu N, Miyajima A. Notch signaling controls hepatoblast differentiation by altering the expression of liver-enriched transcription factors. J Cell Sci 2004;117(Pt 15):3165-3174

65 Yanai M, Tatsumi N, Hasunuma N, Katsu K, Endo F, Yokouchi Y. FGF signaling segregates biliary cell-lineage from chick hepatoblasts cooperatively with BMP4 and ECM components in vitro. Dev Dyn 2008;237(5):1268-1283

66 Hofmann JJ, Zovein AC, Koh H, Radtke F, Weinmaster G, IruelaArispe ML. Jagged1 in the portal vein mesenchyme regulates intrahepatic bile duct development: insights into Alagille syndrome. Development 2010;137(23):4061-4072

67 McDaniell R, Warthen DM, Sanchez-Lara PA, et al. NOTCH2 mutations cause Alagille syndrome, a heterogeneous disorder of the notch signaling pathway. Am J Hum Genet 2006;79(1):169-173

68 McCright B, Lozier J, Gridley T. A mouse model of Alagille syndrome: Notch2 as a genetic modifier of Jag1 haploinsufficiency. Development 2002;129(4):1075-1082

69 Li L, Krantz ID, Deng Y, et al. Alagille syndrome is caused by mutations in human Jagged1, which encodes a ligand for Notch1. Nat Genet 1997;16(3):243-251

70 Oda T, Elkahloun AG, Pike BL, et al. Mutations in the human Jagged1 gene are responsible for Alagille syndrome. Nat Genet 1997;16(3):235-242

71 Geisler F, Nagl F, Mazur PK, et al. Liver-specific inactivation of Notch2, but not Notch1, compromises intrahepatic bile duct development in mice. Hepatology 2008;48(2):607-616

72 Lorent K, Yeo SY, Oda T, et al. Inhibition of Jagged-mediated Notch signaling disrupts zebrafish biliary development and generates multi-organ defects compatible with an Alagille syndrome phenocopy. Development 2004;131(22):5753-5766

73 Hussain SZ, Sneddon T, Tan X, Micsenyi A, Michalopoulos GK, Monga SP. Wnt impacts growth and differentiation in ex vivo liver development. Exp Cell Res 2004;292(1):157-169

74 Choi TY, Ninov N, Stainier DY, Shin D. Extensive conversion of hepatic biliary epithelial cells to hepatocytes after near total loss of hepatocytes in zebrafish. Gastroenterology 2014;146(3):776-788

$75 \mathrm{He}$ J, Lu H, Zou Q Luo L. Regeneration of liver after extreme hepatocyte loss occurs mainly via biliary transdifferentiation in zebrafish. Gastroenterology 2014;146(3):789-800.e8

76 Zajicek G, Oren R, Weinreb M Jr. The streaming liver. Liver 1985; 5(6):293-300

77 Malato Y, Naqvi S, Schürmann N, et al. Fate tracing of mature hepatocytes in mouse liver homeostasis and regeneration. J Clin Invest 2011;121(12):4850-4860 
78 Español-Suñer R, Carpentier R, Van Hul N, et al. Liver progenitor cells yield functional hepatocytes in response to chronic liver injury in mice. Gastroenterology 2012;143(6):1564-1575.e7

79 Higgins GM, Anderson RM. Experimental pathology of the liver I. Restoration of the liver of the white rat following partial surgical removal. Arch Pathol (Chic) 1931;12:186-202

80 Farber E. Similarities in the sequence of early histological changes induced in the liver of the rat by ethionine, 2-acetylaminofluorene, and 3'-methyl-4-dimethylaminoazobenzene. Cancer Res 1956;16(2):142-148

81 Fausto N. Liver regeneration and repair: hepatocytes, progenitor cells, and stem cells. Hepatology 2004;39(6):1477-1487

82 Duncan AW, Dorrell C, Grompe M. Stem cells and liver regeneration. Gastroenterology 2009;137(2):466-481

83 Preisegger KH, Factor VM, Fuchsbichler A, Stumptner C, Denk H, Thorgeirsson SS. Atypical ductular proliferation and its inhibition by transforming growth factor beta1 in the 3,5-diethoxycarbonyl-1,4-dihydrocollidine mouse model for chronic alcoholic liver disease. Lab Invest 1999;79(2):103-109

84 Akhurst B, Croager EJ, Farley-Roche CA, et al. A modified choline-deficient, ethionine-supplemented diet protocol effectively induces oval cells in mouse liver. Hepatology 2001;34(3): 519-522

85 Roskams TA, Theise ND, Balabaud C, et al. Nomenclature of the finer branches of the biliary tree: canals, ductules, and ductular reactions in human livers. Hepatology 2004;39(6):1739-1745

86 Miyajima A, Tanaka M, Itoh T. Stem/progenitor cells in liver development, homeostasis, regeneration, and reprogramming. Cell Stem Cell 2014;14(5):561-574

87 Van Hul NK, Abarca-Quinones J, Sempoux C, Horsmans Y, Leclercq IA. Relation between liver progenitor cell expansion and extracellular matrix deposition in a CDE-induced murine model of chronic liver injury. Hepatology 2009;49(5):1625-1635

88 Lorenzini S, Bird TG, Boulter L, et al. Characterisation of a stereotypical cellular and extracellular adult liver progenitor cell niche in rodents and diseased human liver. Gut 2010;59(5):645-654

89 Kuramitsu K, Sverdlov DY, Liu SB, et al. Failure of fibrotic liver regeneration in mice is linked to a severe fibrogenic response driven by hepatic progenitor cell activation. Am J Pathol 2013; 183(1):182-194

90 Hayner NT, Braun L, Yaswen P, Brooks M, Fausto N. Isozyme profiles of oval cells, parenchymal cells, and biliary cells isolated by centrifugal elutriation from normal and preneoplastic livers. Cancer Res 1984;44(1):332-338

91 Yaswen P, Hayner NT, Fausto N. Isolation of oval cells by centrifugal elutriation and comparison with other cell types purified from normal and preneoplastic livers. Cancer Res 1984;44(1):324-331

92 Sirica AE, Mathis GA, Sano N, Elmore LW. Isolation, culture, and transplantation of intrahepatic biliary epithelial cells and oval cells. Pathobiology 1990;58(1):44-64

93 Theise ND, Saxena R, Portmann BC, et al. The canals of Hering and hepatic stem cells in humans. Hepatology 1999;30(6):1425-1433

94 Lanzoni G, Cardinale V, Carpino G. The hepatic, biliary, and pancreatic network of stem/progenitor cell niches in humans: a new reference frame for disease and regeneration. Hepatology 2016;64(1):277-286

95 Wang B, Zhao L, Fish M, Logan CY, Nusse R. Self-renewing diploid Axin2(+) cells fuel homeostatic renewal of the liver. Nature 2015; 524(7564):180-185

96 Kopp JL, Grompe M, Sander M. Stem cells versus plasticity in liver and pancreas regeneration. Nat Cell Biol 2016;18(3):238-245

97 Reid LM. Stem/progenitor cells and reprogramming (plasticity) mechanisms in liver, biliary tree, and pancreas. Hepatology 2016; 64(1):4-7

98 Huch M, Dollé L. The plastic cellular states of liver cells: are EpCAM and Lgr5 fit for purpose? Hepatology 2016;64(2): 652-662
99 Sackett SD, Li Z, Hurtt R, et al. Foxl1 is a marker of bipotential hepatic progenitor cells in mice. Hepatology 2009;49(3):920-929

100 Furuyama K, Kawaguchi Y, Akiyama H, et al. Continuous cell supply from a Sox9-expressing progenitor zone in adult liver, exocrine pancreas and intestine. Nat Genet 2011;43(1):34-41

101 Huch M, Dorrell C, Boj SF, et al. In vitro expansion of single Lgr5+ liver stem cells induced by Wnt-driven regeneration. Nature 2013;494(7436):247-250

102 Rodrigo-Torres D, Affò S, Coll M, et al. The biliary epithelium gives rise to liver progenitor cells. Hepatology 2014;60(4):1367-1377

103 Schaub JR, Malato Y, Gormond C, Willenbring H. Evidence against a stem cell origin of new hepatocytes in a common mouse model of chronic liver injury. Cell Reports 2014;8(4):933-939

104 Tarlow BD, Finegold MJ, Grompe M. Clonal tracing of Sox9+ liver progenitors in mouse oval cell injury. Hepatology 2014;60(1): 278-289

105 Yanger K, Knigin D, Zong Y, et al. Adult hepatocytes are generated by self-duplication rather than stem cell differentiation. Cell Stem Cell 2014;15(3):340-349

106 Lu WY, Bird TG, Boulter L, et al. Hepatic progenitor cells of biliary origin with liver repopulation capacity. Nat Cell Biol 2015;17(8): 971-983

107 Kamimoto K, Kaneko K, Kok CY, Okada H, Miyajima A, Itoh T. Heterogeneity and stochastic growth regulation of biliary epithelial cells dictate dynamic epithelial tissue remodeling. eLife 2016;5:e15034

108 Lemaigre FP. Determining the fate of hepatic cells by lineage tracing: facts and pitfalls. Hepatology 2015;61(6):2100-2103

109 Reid LM. Paradoxes in studies of liver regeneration: relevance of the parable of the blind men and the elephant. Hepatology 2015; 62(2):330-333

110 Takayama K, Mitani S, Nagamoto Y, et al. Laminin 411 and 511 promote the cholangiocyte differentiation of human induced pluripotent stem cells. Biochem Biophys Res Commun 2016; 474(1):91-96

111 Clevers H. Modeling development and disease with organoids. Cell 2016;165(7):1586-1597

112 Lancaster MA, Knoblich JA. Organogenesis in a dish: modeling development and disease using organoid technologies. Science 2014;345(6194):1247125

113 Dedhia PH, Bertaux-Skeirik N, Zavros Y, Spence JR. Organoid models of human gastrointestinal development and disease. Gastroenterology 2016;150(5):1098-1112

114 Huch M, Gehart H, van Boxtel R, et al. Long-term culture of genome-stable bipotent stem cells from adult human liver. Cell 2015;160(1-2):299-312

115 Takebe T, Sekine K, Enomura M, et al. Vascularized and functional human liver from an iPSC-derived organ bud transplant. Nature 2013;499(7459):481-484

116 Michalopoulos GK, Bowen WC, Mulè K, Stolz DB. Histological organization in hepatocyte organoid cultures. Am J Pathol 2001; 159(5):1877-1887

117 Shin S, Walton G, Aoki R, et al. Foxl1-Cre-marked adult hepatic progenitors have clonogenic and bilineage differentiation potential. Genes Dev 2011;25(11):1185-1192

118 Tanimizu N, Miyajima A, Mostov KE. Liver progenitor cells develop cholangiocyte-type epithelial polarity in three-dimensional culture. Mol Biol Cell 2007;18(4):1472-1479

119 Kido T, Koui Y, Suzuki K, et al. CPM is a useful cell surface marker to isolate expandable bi-potential liver progenitor cells derived from human iPS cells. Stem Cell Rep 2015;5(4):508-515

120 Lugli N, Kamileri I, Keogh A, et al. R-spondin 1 and noggin facilitate expansion of resident stem cells from non-damaged gallbladders. EMBO Rep 2016;17(5):769-779

121 Yu B, He ZY, You P, et al. Reprogramming fibroblasts into bipotential hepatic stem cells by defined factors. Cell Stem Cell 2013;13(3):328-340 